\title{
Surface Effects on a Coated Fiber with an Imperfect Interface Subjected to Plane Compressional Wave
}

\author{
Dongxia Lei, Lizhen Wang, Zhiying Ou* \\ School of Science, Lanzhou University of Technology, Lanzhou, China \\ Email address: \\ zhiyingou@163.com (Zhiying Ou) \\ *Corresponding author
}

\section{To cite this article:}

Dongxia Lei, Lizhen Wang, Zhiying Ou. Surface Effects on a Coated Fiber with an Imperfect Interface Subjected to Plane Compressional Wave. International Journal of Applied Mathematics and Theoretical Physics. Vol. 5, No. 2, 2019, pp. 38-44. doi: 10.11648/j.ijamtp.20190502.11

Received: April 29, 2019; Accepted: May 21, 2019; Published: June 20, 2019

\begin{abstract}
With the rapid development of nanotechnology, nano-components and nano-materials will be widely concerned and applied. At the nano-scale, due to the obvious increase the ratio of surface area to the volume effect and surface effect of nano-components and nano-materials are significant, making their mechanical properties significantly different from the material properties under the macroscopic conditions. And in the practical cases, the interface is not always perfect and smooth, they always have a certain form of defects. Therefore, the wave function expansion method is used in the analytical solutions of dynamic stress concentration factor (DSCF) around a coated fiber with an imperfect interface at nano-scale. The stress boundary conditions on the interface are obtained by using the generalized Young-Laplace equation and the imperfect displacement boundary conditions on the interface are modeled by a spring model. Considering the effects of surface and spring model, the influence of spring stiffness, the number of incident wave and the surface effects on the DSCF are analyzed. The results show that the frequency of incident wave, the spring stiffness and the surface energy have significant effects on the dynamic stress concentration distributions of the nano-sized coated fiber. The smaller the spring coefficient is, the stronger the interface imperfection is, and the stronger the stress concentration at the boundary is. When the spring coefficient reaches a certain value, it is almost close to the dynamic stress value under the ideal interface. The DSCF are obviously different under different incident wave frequencies.
\end{abstract}

Keywords: Scattering, Plane Compressional Wave, Imperfect Interface, Surface Effect, Dynamic Stress Concentration Factor

\section{Introduction}

The scattering of elastic waves by a single scatter with uniform curvature (e.g., cylindrical and spherical) embedded in an elastic matrix is an important topic in wave motion, due to the significant role of scattering in understanding various wave propagation phenomena in engineering materials and structures. In most fiber-reinforced materials, coated-layer fiber is also widely employed to improve the bonding strength between the fiber and the matrix. Thus, many studies have focused on elastic wave scattering from layered cylindrical components (e.g., clad rods, wires, coated fibers, etc.) encased in an elastic matrix. For example, a theoretical method for studying the dynamic response of a circular lined tunnel with an imperfectly bonded interface subjected to plane P-waves is presented. The wave function expansion method was used and the imperfect interface was modeled with a spring model in the paper [1, 2]. Using the wave function expansion method and conformal transformation method, Fang et al. [3-6] derived the dynamic stress around a non-circular tunnel with imperfect interface subjected to anti-plane shear waves, $\mathrm{P}$ waves and SV waves. The stiffness parameter and viscosity coefficient are used to take into account the visco-elastic characteristic of the interface. To improve the strength of tunnels, large values of tangential stiffness parameter and viscosity coefficient of interface are proposed. The effect of viscosity coefficient on the dynamic stress in the region of high frequency is smaller than that in the region of low frequency. The solving method and conclusions can provide significant references in the design of underground tunnels, especially those in the earthquake prone. Qin et al. [7] analyzed three kinds of resonant modes of a single layered circular elastic cylinder embedded in the elastic medium. Stamos et al. [8] studied the 3-D seismic response of long 
lined tunnels in half space with a special boundary element method. Esmaeili et al. [9] investigated the dynamic response of a lined circular tunnel to plane harmonic waves by using hybrid boundary and finite element methods. Valier-Brasier et al. [10] considered that the contacting solid half-spaces are connected by tangential and normal springs, and the mechanical boundary conditions are described. Laser ultrasonic techniques allow the remote analysis of adhesion mechanisms at imperfect interfaces up to $\mathrm{GHz}$ frequencies. However, the sensitivity of interface waves to the properties of the contact is not very well known. Wang et al. [11] investigated the response of a tunnel in double-layer rocks subjected to harmonic $\mathrm{P}$ and $\mathrm{S}$ waves. Huang et al. [12] studied the scattering of an elastic wave obliquely incident on a multilayered cylinder in a solid. Hasheminejad et al. [13] employed exact treatment based on the inherent background coefficients that describe the background amplitudes in the scattered field. Shindo et al. [14] studied with the scattering of antiplane shear waves in a metal matrix composite reinforced by fibers with interracial layers. Li et al. [15] studied the scattering and dynamic stress concentration in fiber-reinforced composite with interfaces.

Owing to the increasing ratio of surface area to volume, surface/interface energy has a significant effect on the matrials properties of nanostructured materials. Thus the nanostructured materials display some interesting physical, optical, and dynamical properties distinct from their macroscopic counterparts [16]. To incorporate the influence of surface/interface energy in the continuum theory of solids, the surface elasticity model developed by Gurtin and Murdoch [17] and Gurtin et al. [18] has been widely used in analyzing various dynamical phenomena. Wang et al. [19] considered the diffraction of P-wave by a nano-sized circular hole. Based on the surface elasticity theory, Wang [20] analyzed the diffraction of plane harmonic compressional wave $\mathrm{P}$ wave by a spherical cavity. And Wang [21] investigated the multiple diffraction of plane harmonic compressional waves by two nanosized circular cylindrical holes embedded in an elastic solid. Ru et al. [22] described the diffraction of elastic waves and the stress concentration near a cylindrical nano-inclusion with surface effect. Ou et al. [23] investigated the multiple scattering of plane compressional waves by two cylindrical fibers with interface effects. $\mathrm{Ru}$ et al. [24] considers the multiple scattering of vertical shear waves (SV waves) by a cluster of nano-sized cylindrical holes. Ou et al. [25] studied the scattering of plane compressional and shear waves by a single nano-sized coated fiber embedded in an elastic matrix using the method of wave function expansion. Their results demonstrated the considerable importance of surface effects on the dynamic stress concentration. As the discontinuing of stresses and displacements at the interface, there is few work addressing the wave scattering by of the nano-sized coated fiber with an imperfect interface.

In this paper, we examined the scattering of an elastic wave by a coated layer with an imperfect interface. Using the method of wave function expansion, the elastic dynamic fields were derived and numerically calculated and the dynamic stress concentration factors (DSCF) along the interface were obtained. Our results showed that the influence of the interface stresses on the DSCF becomes significant when the radius of the inclusion is reduced to the nanometer scale. Meanwhile the results indicated that the variation in the stiffness of the imperfect interface has much influence on the distribution of DSCF in the matrix and shell.

\section{Model Analysis and Formulations}

Based on the surface elasticity, we consider the diffraction of an elastic cylindrical coated fiber embedded in an infinite isotropic matrix, in Figure 1. And in the work, the spring model is adopted to model the imperfect interface. The outer radius of the model is $a$ and its inner radius is $b$ and the center is $O$. A harmonically time-varying incident plane $\mathrm{P}$ wave propagates in the matrix and meets perpendicularly the shell. The elastic constants and the densities of the matrix, shell and core are $\lambda^{(j)}, \mu^{(j)}$ and $\rho^{(j)}(j=1,2,3)$, the superscripts 1, 2 and 3 indicate the quantities associated with the core, coated layer, and matrix, respectively.

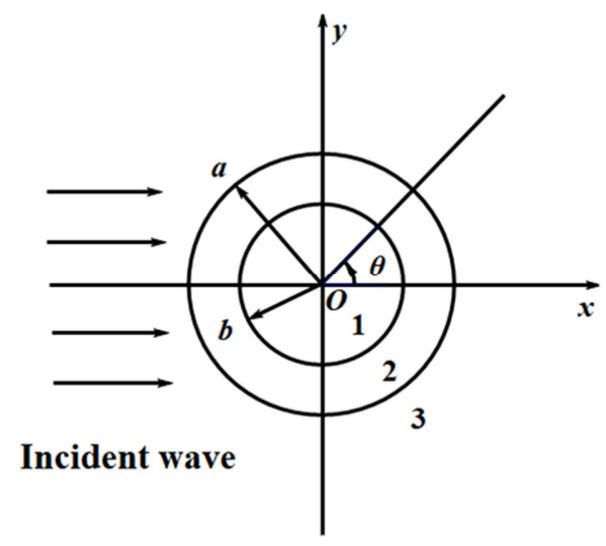

Figure 1. Cross section of scattering of incident wave by a cylindrical coated fiber with an imperfect interface.

Assuming a harmonically plane $\mathrm{P}$ wave propagating in the positive $\mathrm{x}$-direction, the displacement potential of the incident wave in the cylindrical coordinate $(r, \theta)$ can be written as

$$
\varphi^{\mathrm{inc}}(r, \theta)=\varphi_{0} \sum_{n=0}^{\infty} \varepsilon_{n} i^{n} J_{n}\left(\alpha_{3} r\right) \cos n \theta
$$

Where $\varphi_{0}$ is the amplitude of the incident wave, $\varepsilon_{n}$ is the Neumann factor ( $\varepsilon_{n}=1$ for $n=0$ and $\varepsilon_{n}=2$ for $n>0$ ), $\alpha_{3}=\omega / c_{p}^{(3)}$ is the $\mathrm{P}$ wave number of the matrix and $c_{p}^{(3)}=\sqrt{\left(\lambda^{(3)}+2 \mu^{(3)}\right) / \rho^{(3)}}$ is the wave velocity, $J_{n}(x)$ is the nth order Bessel function of the first kind.

When it impinges on the coated fiber, the $\mathrm{P}$ wave and SV wave are scattered back from the interface $r=a$, and the corresponding displacement potentials of the scattered wave are given by 


$$
\begin{aligned}
& \varphi^{\mathrm{sca}}(r, \theta)=\varphi_{0} \sum_{n=0}^{\infty} A_{n 3} H_{n}^{(1)}\left(\alpha_{3} r\right) \cos n \theta, \\
& \psi^{\mathrm{sca}}(r, \theta)=\varphi_{0} \sum_{n=0}^{\infty} B_{n 3} H_{n}^{(1)}\left(\beta_{3} r\right) \sin n \theta
\end{aligned}
$$

where $A_{n 3}$ and $B_{n 3}$ are unknown coefficients in the matrix, $H_{n}^{(1)}(x)$ is the nth order Hankel function of the first kind, $\beta_{3}=\omega / c_{s}^{(3)}$ is the $\mathrm{SV}$ wave number of the matrix and $c_{s}^{(3)}=\sqrt{\mu^{(3)} / \rho^{(3)}}$ is the shear wave velocity.

So the total waves in the matrix are determined by the superposition of the incident waves and scattered waves

$$
\begin{gathered}
\varphi^{(3)}(r, \theta)=\varphi^{\mathrm{inc}}(r, \theta)+\varphi^{\mathrm{sca}}(r, \theta), \\
\psi^{(3)}(r, \theta)=\psi^{\mathrm{sca}}(r, \theta),
\end{gathered}
$$

The standing compressional and shear waves reverberating inside the dissipative interphase are described as

$$
\begin{aligned}
& \varphi^{(2)}(r, \theta)=\varphi_{0} \sum_{n=0}^{\infty}\left[A_{n 2} J_{n}\left(\alpha_{2} r\right)+C_{n 2} H_{n}^{(2)}\left(\alpha_{2} r\right)\right] \cos n \theta \\
& \psi^{(2)}(r, \theta)=\varphi_{0} \sum_{n=0}^{\infty}\left[B_{n 2} J_{n}\left(\beta_{2} r\right)+D_{n 2} H_{n}^{(2)}\left(\beta_{2} r\right)\right] \sin n \theta
\end{aligned}
$$

where $A_{n 2}, C_{n 2}, B_{n 2}$ and $D_{n 2}$ are the unknown coefficients of the interphase; $H_{n}^{(2)}(x)$ is the $n$th order Hankel function of the second kind, and $\alpha_{2}=\omega / c_{p}^{(2)}$, $\beta_{2}=\omega / c_{s}^{(2)}$ are the $\mathrm{P}$ wave and $\mathrm{SV}$ wave number of the coated fiber, $c_{p}^{(2)}=\sqrt{\left(\lambda^{(2)}+2 \mu^{(2)}\right) / \rho^{(2)}}$ and $c_{s}^{(2)}=\sqrt{\mu^{(2)} / \rho^{(2)}}$ are the wave velocity of the $\mathrm{P}$ wave and $\mathrm{SV}$ wave, respectively.

Lastly, the refracted waves transmitted into the core can be expressed as

$$
\begin{aligned}
& \varphi^{(1)}(r, \theta)=\varphi_{0} \sum_{n=0}^{\infty} A_{n 1} J_{n}\left(\alpha_{1} r\right) \cos n \theta, \\
& \psi^{(1)}(r, \theta)=\varphi_{0} \sum_{n=0}^{\infty} B_{n 1} J_{n}\left(\beta_{1} r\right) \sin n \theta .
\end{aligned}
$$

where $A_{n 1}, B_{n 1}$ are the unknown coefficients in the core, $J_{n}(x)$ is the $n$th order Bessel function of the first kind, and $\alpha_{1}=\omega / c_{p}^{(1)}, \beta_{1}=\omega / c_{s}^{(1)}$ are the $\mathrm{P}$ wave and $\mathrm{SV}$ wave number of the core, $c_{p}^{(1)}=\sqrt{\left(\lambda^{(1)}+2 \mu^{(1)}\right) / \rho^{(1)}}$ and $c_{s}^{(1)}=\sqrt{\mu^{(1)} / \rho^{(1)}}$ are the wave velocity.
Based on the relations between potentials, stresses and displacements, the displacements in cylindrical coordinates are

$$
\begin{aligned}
& u_{r}=\frac{\partial \varphi}{\partial r}+\frac{1}{r} \frac{\partial \psi}{\partial \theta} \\
& u_{\theta}=\frac{1}{r} \frac{\partial \varphi}{\partial \theta}-\frac{\partial \psi}{\partial r}
\end{aligned}
$$

and the corresponding stresses in the cylindrical coordinates are

$$
\begin{gathered}
\sigma_{r r}=\lambda\left[\frac{\partial^{2} \varphi}{\partial r^{2}}+\frac{1}{r} \frac{\partial \varphi}{\partial r}+\frac{1}{r^{2}} \frac{\partial^{2} \varphi}{\partial \theta^{2}}\right]+2 \mu\left[\frac{\partial^{2} \varphi}{\partial r^{2}}-\frac{1}{r^{2}} \frac{\partial \psi}{\partial \theta}+\frac{1}{r} \frac{\partial^{2} \psi}{\partial r \partial \theta}\right] \\
\sigma_{\theta \theta}=\lambda\left[\frac{\partial^{2} \varphi}{\partial r^{2}}+\frac{1}{r} \frac{\partial \varphi}{\partial r}+\frac{1}{r^{2}} \frac{\partial^{2} \varphi}{\partial \theta^{2}}\right]+2 \mu\left[\frac{1}{r^{2}} \frac{\partial^{2} \varphi}{\partial \theta^{2}}+\frac{1}{r} \frac{\partial \varphi}{\partial r}-\frac{1}{r} \frac{\partial^{2} \psi}{\partial r \partial \theta}+\frac{1}{r^{2}} \frac{\partial \psi}{\partial \theta}\right] \\
\sigma_{r \theta}=\frac{\mu}{r}\left[2 \frac{\partial^{2} \varphi}{\partial r \partial \theta}-\frac{2}{r} \frac{\partial \varphi}{\partial \theta}+\frac{1}{r} \frac{\partial^{2} \psi}{\partial \theta^{2}}-r \frac{\partial^{2} \psi}{\partial r^{2}}+\frac{\partial \psi}{\partial r}\right]
\end{gathered}
$$

where $\lambda$ and $\mu$ are the Lame constants.

Since the time representing the steady state harmonic motions exists in the incident waves and also in all resulting waves, it will be omitted in all subsequent expressions. Substituting Eqs. (3)-(5) into Eqs. (6) and (7), we can obtain the matrix displacements

$$
u_{r 3}=\frac{1}{r} \sum_{n=0}^{\infty}\left[\varepsilon_{n} i^{n} M_{71}^{(1)}\left(\alpha_{3} r\right)+A_{n 3} M_{71}^{(3)}\left(\alpha_{3} r\right)+B_{n 3} M_{72}^{(3)}\left(\beta_{3} r\right)\right] \cos n \theta,
$$

$u_{\theta 3}=-\frac{1}{r} \sum_{n=0}^{\infty}\left[\varepsilon_{n} i^{n} M_{81}^{(1)}\left(\alpha_{3} r\right)+A_{n 3} M_{81}^{(3)}\left(\alpha_{3} r\right)+B_{n 3} M_{82}^{(3)}\left(\beta_{3} r\right)\right] \sin n \theta$,

and the matrix stresses

$\sigma_{r r 3}=\frac{2 \mu^{(3)}}{r^{2}} \sum_{n=0}^{\infty}\left[\varepsilon_{n} i^{n} E_{11}^{(1)}\left(\alpha_{3} r\right)+A_{n 3} E_{11}^{(3)}\left(\alpha_{3} r\right)+B_{n 3} E_{12}^{(3)}\left(\beta_{3} r\right)\right] \cos n \theta,(17)$

$\sigma_{r \theta 3}=\frac{2 \mu^{(3)}}{r^{2}} \sum_{n=0}^{\infty}\left[\varepsilon_{n}{ }^{n} E_{41}^{(1)}\left(\alpha_{3} r\right)+A_{n 3} E_{41}^{(3)}\left(\alpha_{3} r\right)+B_{n 3} E_{42}^{(3)}\left(\beta_{3} r\right)\right] \sin n \theta$,

$\sigma_{\theta \theta 3}=\frac{2 \mu^{(3)}}{r^{2}} \sum_{n=0}^{\infty}\left[\varepsilon_{n} \mathrm{i}^{n} E_{21}^{(1)}\left(\alpha_{3} r\right)+A_{n 3} E_{21}^{(3)}\left(\alpha_{3} r\right)+B_{n 3} E_{22}^{(3)}\left(\beta_{3} r\right)\right] \cos n \theta$

The displacements of the shell

$$
\begin{aligned}
u_{r 2}= & \frac{1}{r} \sum_{n=0}^{\infty}\left[A_{n 2} M_{71}^{(1)}\left(\alpha_{2} r\right)+C_{n 2} M_{71}^{(4)}\left(\alpha_{2} r\right)\right. \\
& \left.+B_{n 2} M_{72}^{(1)}\left(\beta_{2} r\right)+D_{n 2} M_{72}^{(4)}\left(\beta_{2} r\right)\right] \cos n \theta, \\
u_{\theta 2}= & \frac{1}{r} \sum_{n=0}^{\infty}\left[A_{n 2} M_{81}^{(1)}\left(\alpha_{2} r\right)+C_{n 2} M_{81}^{(4)}\left(\alpha_{2} r\right)\right.
\end{aligned}
$$




$$
\left.+B_{n 2} M_{82}^{(1)}\left(\beta_{2} r\right)+D_{n 2} M_{82}^{(4)}\left(\beta_{2} r\right)\right] \sin n \theta,
$$

and the stresses of the shell

$$
\begin{gathered}
\sigma_{r r 2}=\frac{2 \mu^{(2)}}{r^{2}} \sum_{n=0}^{\infty}\left[A_{n 2} E_{11}^{(1)}\left(\alpha_{2} r\right)+C_{n 2} E_{11}^{(4)}\left(\alpha_{2} r\right)\right. \\
\left.+B_{n 2} E_{12}^{(1)}\left(\beta_{2} r\right)+D_{n 2} E_{12}^{(4)}\left(\beta_{2} r\right)\right] \cos n \theta \\
\sigma_{r \theta 2}=\frac{2 \mu^{(2)}}{r^{2}} \sum_{n=0}^{\infty}\left[A_{n 2} E_{41}^{(1)}\left(\alpha_{2} r\right)+C_{n 2} E_{41}^{(4)}\left(\alpha_{2} r\right)\right. \\
\left.+B_{n 2} E_{42}^{(1)}\left(\beta_{2} r\right)+D_{n 2} E_{42}^{(4)}\left(\beta_{2} r\right)\right] \sin n \theta, \\
\sigma_{\theta \theta 2}=\frac{2 \mu^{(2)}}{r^{2}} \sum_{n=0}^{\infty}\left[A_{n 2} E_{21}^{(1)}\left(\alpha_{2} r\right)+C_{n 2} E_{21}^{(4)}\left(\alpha_{2} r\right)\right. \\
\left.+B_{n 2} E_{22}^{(1)}\left(\beta_{2} r\right)+D_{n 2} E_{22}^{(4)}\left(\beta_{2} r\right)\right] \cos n \theta .
\end{gathered}
$$

The displacements of the core

$$
\begin{gathered}
u_{r 1}=\frac{1}{r} \sum_{n=0}^{\infty}\left[A_{n 1} M_{71}^{(1)}\left(\alpha_{1} r\right)+B_{n 1} M_{72}^{(1)}\left(\beta_{1} r\right)\right] \cos n \theta \\
u_{\theta 1}=-\frac{1}{r} \sum_{n=0}^{\infty}\left[A_{n 1} M_{81}^{(1)}\left(\alpha_{1} r\right)+B_{n 1} M_{82}^{(1)}\left(\beta_{1} r\right)\right] \sin n \theta
\end{gathered}
$$

and stresses of the core

$$
\begin{aligned}
& \sigma_{r r 1}=\frac{2 \mu^{(1)}}{r^{2}} \sum_{n=0}^{\infty}\left[A_{n 1} E_{11}^{(1)}\left(\alpha_{1} r\right)+B_{n 1} E_{12}^{(1)}\left(\beta_{1} r\right)\right] \cos n \theta, \\
& \sigma_{r \theta 1}=\frac{2 \mu^{(1)}}{r^{2}} \sum_{n=0}^{\infty}\left[A_{n 1} E_{41}^{(1)}\left(\alpha_{1} r\right)+B_{n 1} E_{42}^{(1)}\left(\beta_{1} r\right)\right] \sin n \theta, \\
& \sigma_{\theta \theta 1}=\frac{2 \mu^{(1)}}{r^{2}} \sum_{n=0}^{\infty}\left[A_{n 1} E_{21}^{(1)}\left(\alpha_{1} r\right)+B_{n 1} E_{22}^{(1)}\left(\beta_{1} r\right)\right] \cos n \theta .
\end{aligned}
$$

where $\mu^{(1)}, \mu^{(2)}, \mu^{(3)}$ are the respective Lame constants for matrix, shell and core and $M_{i j}^{(k)}, E_{i j}^{(k)}$ is the contribution of various waves for stresses and displacements (see Appendix).

Finally, at the interfaces $r=a$ and $r=b$, the model assumes that traction satisfies the Young-Laplace equations (the derivation process is the same as reference [25]) but displacements may be discontinuous across the interface. These interface parameters are assumed to be uniform along the entire length of the interface and the interface model is said to represent a homogeneously imperfect interface. Using the above concept, the boundary conditions at the interface between the core and coated fiber, the fiber and matrix are

$$
\begin{gathered}
u_{r 3}-u_{r 2}=\frac{\sigma_{r r 3}}{k_{r 3}}, u_{\theta 3}-u_{\theta 2}=\frac{\sigma_{r \theta 3}}{k_{\theta 3}}, \\
u_{r 1}-u_{r 2}=\frac{\sigma_{r r 1}}{k_{r 1}}, u_{\theta 1}-u_{\theta 2}=\frac{\sigma_{r \theta 1}}{k_{\theta 1}}, \\
\sigma_{r r 3}-\sigma_{r r 2}=s_{2}\left(1-v_{3}\right) \sigma_{\theta \theta 3}-s_{2} v_{3} \sigma_{r r 3}, \\
\sigma_{r \theta 3}-\sigma_{r \theta 2}=-s_{2}\left(1-v_{3}\right) \frac{\partial \sigma_{\theta \theta 3}}{\partial \theta}+s_{2} v_{3} \frac{\partial \sigma_{r r 3}}{\partial \theta}, \\
\sigma_{r r 1}-\sigma_{r r 2}=s_{1}\left(1-v_{1}\right) \sigma_{\theta \theta 1}-s_{1} v_{1} \sigma_{r r 1}, \\
\sigma_{r \theta 1}-\sigma_{r \theta 2}=-s_{1}\left(1-v_{1}\right) \frac{\partial \sigma_{\theta \theta 1}}{\partial \theta}+s_{1} v_{1} \frac{\partial \sigma_{r r 1}}{\partial \theta}, \\
s_{1}=\frac{2 \mu^{s}+\lambda^{s}}{2 \mu_{2} b}=\eta s_{2}, s_{2}=\frac{2 \mu^{s}+\lambda^{s}}{2 \mu_{2} a}, \eta=\frac{a}{b} .
\end{gathered}
$$

Substituting Eqs. (15)-(29) into the boundary condition Eqs. (30)-(35) on the surface and making use of the orthogonaity of $e^{i n \theta}$, we obtained a set of equations (37)-(44) with the coefficients, the coefficients were determined by truncation, then the elastic scattering fields due to the incident $\mathrm{P}$ wave can be determined.

$$
\begin{gathered}
\varepsilon_{n} \mathrm{i}^{n} M_{71}^{(1)}\left(\alpha_{3} a\right)+A_{n 3} M_{71}^{(3)}\left(\alpha_{3} a\right)+B_{n 3} M_{72}^{(3)}\left(\beta_{3} a\right) \\
-\left(A_{n 2} M_{71}^{(1)}\left(\alpha_{2} a\right)+C_{n 2} M_{71}^{(4)}\left(\alpha_{2} a\right)+B_{n 2} M_{72}^{(1)}\left(\beta_{2} a\right)+D_{n 2} M_{72}^{(4)}\left(\beta_{2} a\right)\right) \\
=\frac{2 \mu^{(3)}}{k_{r 3} a}\left(\varepsilon_{n} \mathrm{i}^{n} E_{11}^{(1)}\left(\alpha_{3} a\right)+A_{n 3} E_{11}^{(3)}\left(\alpha_{3} a\right)+B_{n 3} E_{12}^{(3)}\left(\beta_{3} a\right)\right), \\
-\left(\varepsilon_{n} \mathrm{i}^{n} M_{81}^{(1)}\left(\alpha_{3} a\right)+A_{n 3} M_{81}^{(3)}\left(\alpha_{3} a\right)+B_{n 3} M_{82}^{(3)}\left(\beta_{3} a\right)\right) \\
+\left(A_{n 2} M_{81}^{(1)}\left(\alpha_{2} a\right)+C_{n 2} M_{81}^{(4)}\left(\alpha_{2} a\right)+B_{n 2} M_{82}^{(1)}\left(\beta_{2} a\right)+D_{n 2} M_{82}^{(4)}\left(\beta_{2} a\right)\right) \\
=\frac{2 \mu^{(3)}}{k_{\theta 3} a}\left(\varepsilon_{n} \mathrm{i}^{n} E_{41}^{(1)}\left(\alpha_{3} a\right)+A_{n 3} E_{41}^{(3)}\left(\alpha_{3} a\right)+B_{n 3} E_{42}^{(3)}\left(\beta_{3} a\right)\right), \\
A_{n 1} M_{71}^{(1)}\left(\alpha_{1} b\right)+B_{n 1} M_{72}^{(1)}\left(\beta_{1} b\right) \\
-\left(A_{n 2} M_{71}^{(1)}\left(\alpha_{2} b\right)+C_{n 2} M_{71}^{(4)}\left(\alpha_{2} b\right)+B_{n 2} M_{72}^{(1)}\left(\beta_{2} b\right)+D_{n 2} M_{72}^{(4)}\left(\beta_{2} b\right)\right) \\
=\frac{2 \mu^{(1)}}{k_{r 1} b}\left(A_{n 1} E_{11}^{(1)}\left(\alpha_{1} b\right)+B_{n 1} E_{12}^{(1)}\left(\beta_{1} b\right)\right), \\
-\left(A_{n 1} M_{81}^{(1)}\left(\alpha_{1} b\right)+B_{n 1} M_{82}^{(1)}\left(\beta_{1} b\right)\right) \\
+\left(A_{n 2} M_{81}^{(1)}\left(\alpha_{2} b\right)+C_{n 2} M_{81}^{(4)}\left(\alpha_{2} b\right)+B_{n 2} M_{82}^{(1)}\left(\beta_{2} b\right)+D_{n 2} M_{82}^{(4)}\left(\beta_{2} b\right)\right) \\
=\frac{2 \mu^{(1)}}{k_{\theta 1} b}\left(A_{n 1} E_{11}^{(1)}\left(\alpha_{1} b\right)+B_{n 1} E_{12}^{(1)}\left(\beta_{1} b\right)\right), \\
\varepsilon_{n} \mathrm{i}^{n} E_{11}^{(1)}\left(\alpha_{3} a\right)+A_{n 3} E_{11}^{(3)}\left(\alpha_{3} a\right)+B_{n 3} E_{12}^{(3)}\left(\beta_{3} a\right)
\end{gathered}
$$




$$
\begin{gathered}
-\frac{\mu^{(2)}}{\mu^{(3)}}\left(A_{n 2} E_{11}^{(1)}\left(\alpha_{2} a\right)+C_{n 2} E_{11}^{(4)}\left(\alpha_{2} a\right)+B_{n 2} E_{12}^{(1)}\left(\beta_{2} a\right)+D_{n 2} E_{12}^{(4)}\left(\beta_{2} a\right)\right) \\
=s_{2}\left(1-v_{3}\right)\left(\varepsilon_{n} \mathrm{i}^{n} E_{21}^{(1)}\left(\alpha_{3} a\right)+A_{n 3} E_{21}^{(3)}\left(\alpha_{3} a\right)+B_{n 3} E_{22}^{(3)}\left(\beta_{3} a\right)\right) \\
-s_{2} v_{3}\left(\varepsilon_{n} \mathrm{i}^{n} E_{11}^{(1)}\left(\alpha_{3} a\right)+A_{n 3} E_{11}^{(3)}\left(\alpha_{3} a\right)+B_{n 3} E_{12}^{(3)}\left(\beta_{3} a\right)\right), \quad(41) \\
\varepsilon_{n} \mathrm{i}^{n} E_{41}^{(1)}\left(\alpha_{3} a\right)+A_{n 3} E_{41}^{(3)}\left(\alpha_{3} a\right)+B_{n 3} E_{42}^{(3)}\left(\beta_{3} a\right) \\
-\frac{\mu^{(2)}}{\mu^{(3)}}\left(A_{n 2} E_{41}^{(1)}\left(\alpha_{2} a\right)+C_{n 2} E_{41}^{(4)}\left(\alpha_{2} a\right)+B_{n 2} E_{42}^{(1)}\left(\beta_{2} a\right)+D_{n 2} E_{42}^{(4)}\left(\beta_{2} a\right)\right) \\
=n s_{2}\left(1-v_{3}\right)\left(\varepsilon_{n} \mathrm{i}^{n} E_{21}^{(1)}\left(\alpha_{3} a\right)+A_{n 3} E_{21}^{(3)}\left(\alpha_{3} a\right)+B_{n 3} E_{22}^{(3)}\left(\beta_{3} a\right)\right) \\
-n s_{2} v_{3}\left(\varepsilon_{n} \mathrm{i}^{n} E_{11}^{(1)}\left(\alpha_{3} a\right)+A_{n 3} E_{11}^{(3)}\left(\alpha_{3} a\right)+B_{n 3} E_{12}^{(3)}\left(\beta_{3} a\right)\right), \quad(42) \\
-n s_{1} v_{1}\left(A_{n 1} E_{11}^{(1)}\left(\alpha_{1} b\right)+B_{n 1} E_{12}^{(1)}\left(\beta_{1} b\right)\right) \\
-\frac{\mu^{(2)} E_{11}^{(1)}\left(\alpha_{1} b\right)+B_{n 1} E_{12}^{(1)}\left(\beta_{1} b\right)}{\mu^{(1)}}\left(A_{n 2} E_{41}^{(1)}\left(\alpha_{2} b\right)+C_{n 2} E_{41}^{(4)}\left(\alpha_{2} b\right)+B_{n 2} E_{42}^{(1)}\left(\beta_{2} b\right)+D_{n 2} E_{42}^{(4)}\left(\beta_{2} b\right)\right) \\
-\frac{\mu^{(2)}}{\mu^{(1)}}\left(A_{n 2} E_{11}^{(1)}\left(\alpha_{2} b\right)+C_{n 2} E_{11}^{(4)}\left(\alpha_{2} b\right)+B_{n 2} E_{12}^{(1)}\left(\beta_{2} b\right)+D_{n 2} E_{12}^{(4)}\left(\beta_{2} b\right)\right) \\
=s_{1} E_{41}^{(1)}\left(\alpha_{1} b\right)+B_{n 1} E_{42}^{(1)}\left(\beta_{1} b\right) \\
s_{1} v_{1}\left(A_{n 1} E_{11}^{(1)}\left(\alpha_{1} b\right)+B_{n 1} E_{12}^{(1)}\left(\beta_{1} b\right)\right), \\
\left(1-v_{1}\right)\left(A_{n 1} E_{21}^{(1)}\left(\alpha_{1} b\right)+B_{n 1} E_{22}^{(1)}\left(\beta_{1} b\right)\right)
\end{gathered}
$$

\section{Results and Discussion}

We define the dynamic stress concentration factor (DSCF) as

$$
\mathrm{DSCF}=\left|\frac{\sigma_{\theta \theta 3}}{\sigma_{0}}\right|
$$

where $\sigma_{\theta \theta 3}$ is the bulk stress of the matrix material at interface, $\sigma_{0}=\mu^{(3)} \beta_{3}^{2} \varphi_{0}$ is the stress intensity in the propagation direction of $\mathrm{P}$ wave.

In this paper, through comparing our results with the published results for the special cases under low frequency incident waves to confirm its correctness. The Poisson ratio is assumed to be $v=0.3$ for all calculation. In Figure 2, let $s_{2}=0, k=\infty$, the present nanosized coated fiber reduces to the classical problem without an imperfect interface. The results are in good agreement with those presented in the reference [25].

To make the follow calculations tractable, we assumed that the properties of the core and matrix were identical, but different from those of coating.

\subsection{The Effects of a Low Frequency Incident Wave with $\alpha_{3} a=0.2$}

When the frequency of incident wave is low in Figure 2 and Figure 3, with the increasing of $s_{2}$, the DSCF also increases around $\theta= \pm \pi / 2$; but with the increasing of spring stiffness, the DSCF decreases around $\theta= \pm \pi / 2$. The results also indicate that DSCF at $\theta= \pm \pi / 2$ is maximum. In particular, the DSCF at $\theta=0$ and $\theta= \pm \pi$ is larger in Figure 3, which is quite different from the results of perfect interface that DSCF is always small $[1,2]$.

\subsection{The Effects of a High Frequency Incident Wave $\alpha_{3} a=\pi$}

When the incident wave's frequency is high, the distribution of DSCF is complicated in Figure 4 and Figure 5. At the boundary of matrix and coated layer, the maximum of DSCF is different. And in this case, the magnitude of the DSCF was generally lower than that of a low frequency incident wave, it indicated that the incident wave's frequency has different effects on the dynamic response.

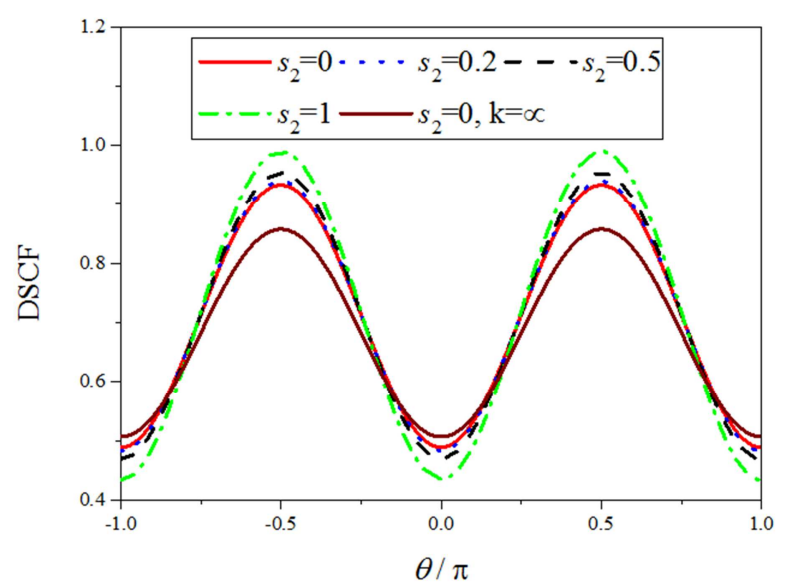

Figure 2. Distribution of DSCF along interface $r=a$ with various values of $s_{2}$ under $\mu^{(2)} / \mu^{(3)}=3$ and $\alpha_{2} / \alpha_{3}=1.5$.

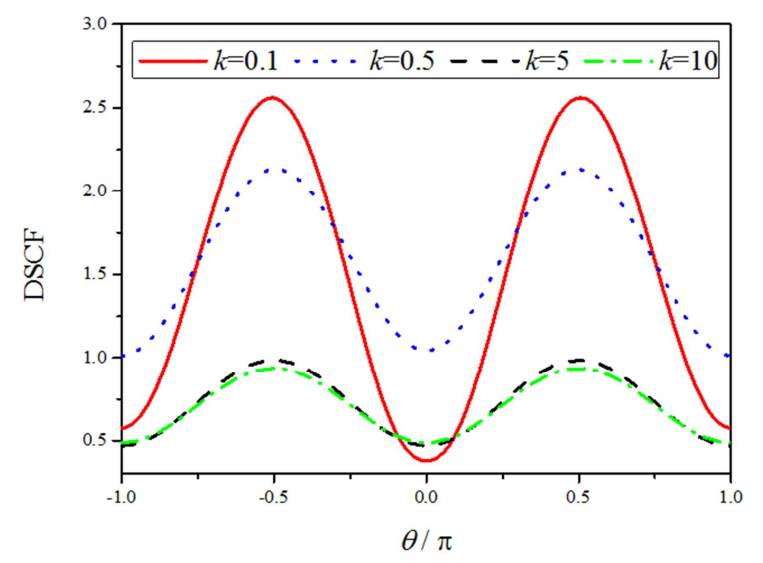

Figure 3. Distribution of DSCF along interface $r=a$ with various values of $k$ under $\mu^{(2)} / \mu^{(3)}=3$ and $\alpha_{2} / \alpha_{3}=1.5$. 


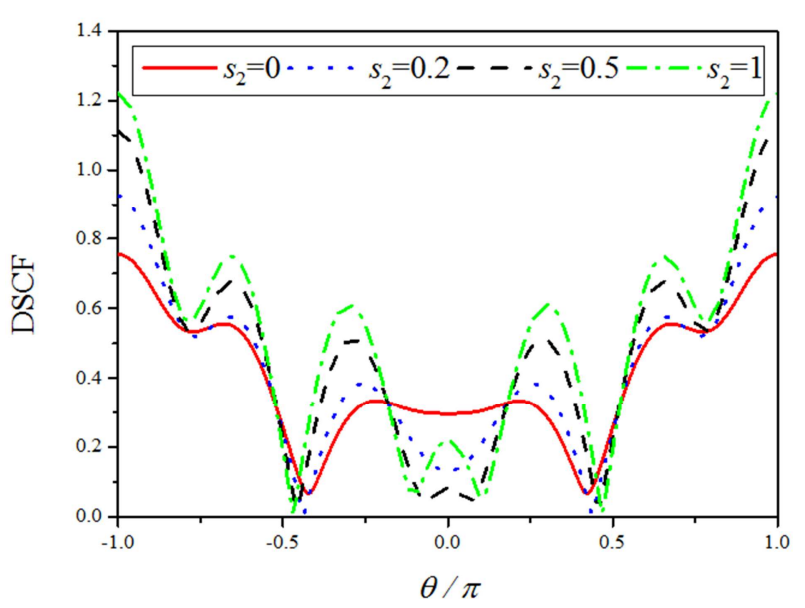

Figure 4. Distribution of DSCF along interface $r=a$ with various values of $s_{2}$ under $\mu^{(2)} / \mu^{(3)}=3$ and $\alpha_{2} / \alpha_{3}=1.5$.

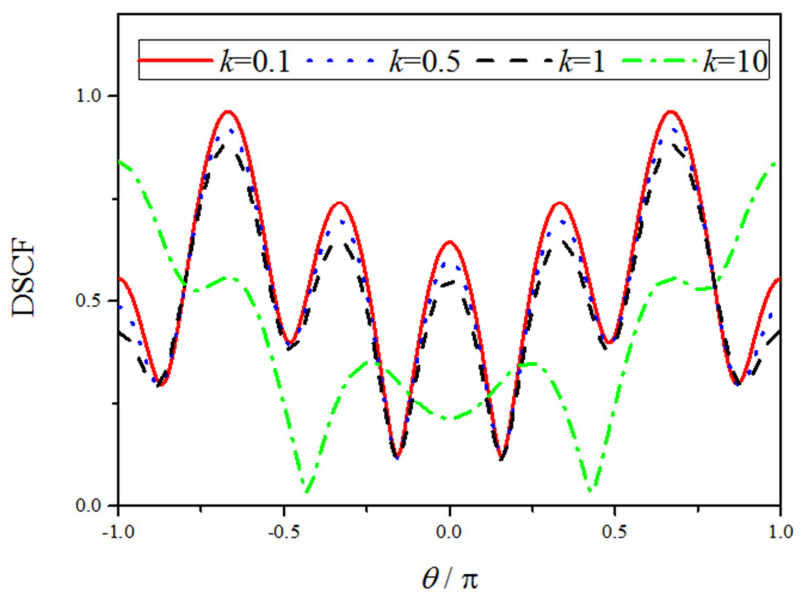

Figure 5. Distribution of DSCF along interface $r=a$ with various values of $k$ under $\mu^{(2)} / \mu^{(3)}=3$ and $\alpha_{2} / \alpha_{3}=1.5$.

\section{Conclusion}

The main work of this paper is to introduce the concept of surface effect on the basis of classical elastic theory. The influence of the interface imperfections and surface effects on the DSCF of a coated fiber subjected to plane incident $P$ wave was studied by using the wave function expansion method and the spring model for the imperfect interface. The distribution of DSCF around the coated fiber with the change of the frequency, spring stiffness and the interface parameter were presented.

The results show that increasing $s_{2}$ leads to a increasing DSCF and increasing spring stiffness leads to a decreasing DSCF. The smaller the spring coefficient is, the stronger the stress concentration degree is, and when it reaches a certain value, the influence of interface imperfection basically disappears, almost approaching the ideal interface. But the DSCF of the coated fiber in the high frequency incident wave is complicated. As the frequency increases, diffraction will occur, and at this frequency diffraction plays a dominant role, making the dynamic stress concentration factor become very irregular. Thus, the influence of an imperfect interface and the surface energy has a pronounced effect on the DSCF when the radius of the coated fiber reduces to the nano-scale.

The solving method and conclusions can provide significant references in the research of surface theory, especially those imperfect interface.

\section{Acknowledgements}

The supports from the National Natural Science Foundation (Grant No. 11362009 and No. 11862014) are acknowledged.

\section{References}

[1] Yi, C. P., Zhang, P., Johansson, D. and Nyberg, U. (2014) Dynamic response of a circular lined tunnel with an imperfect interface subjected to cylindrical P-waves. Computers and Geotechnics, 55, 165-171.

[2] Yi, C. P., Lu, W. B., Zhang, P., Johansson, D. and Nyberg, U. (2016) Effect of imperfect interface on the dynamic response of a circular lined tunnel impacted by plane P-waves. Tunneling and Underground Space Technology, 51, 68-74.

[3] Fang, X. Q., Huang, H. C. and Liu, J. X. (2016) Predicting the crack opening displacements of a partially debonded pipeline in rock mass under $P$ waves. Engineering Materials and Structures, 39, 886-895.

[4] Fang, X. Q., Jin, H. X., Liu, J. X. and Huang, M. J. (2016) Imperfect bonding effect on dynamic response of a non-circular lined tunnel subjected to shear waves. Tunnelling and Underground Space Technology, 56, 226-231.

[5] Fang, X. Q. and Jin, H. X. (2016) Visco-elastic imperfect bonding effect on dynamic response of a non-circular lined tunnel subjected to $\mathrm{P}$ and SV waves. Soil Dynamics and Earthquake Engineering, 88, 1-7.

[6] Fang, X. Q. and Jin, H. X. (2017) Dynamic response of a non-circular lined tunnel with visco-elastic imperfect interface in the saturated poroelastic medium. Computers and Geotechnics, 83, 98-105.

[7] Qin, B., Chen, J. J. and Cheng, J. C. (2005) Local resonant characteristics of a layered cylinder embedded in the elastic medium. Chinese Physics, 14, 2522-2528.

[8] Stamos, A. A. and Beskos, D. E. (1996) 3-D seismic response analysis of long lined tunnels in half-space. Soil Dynamics and Earthquake Engineering, 15, 111-118.

[9] Esmaeili M., Vahdani S. and Noorzad, A. (2006) Dynamic response of lined circular tunnel to plane harmonic waves. Tunneling and Underground Space Technology, 21, 511-519.

[10] Valier-Brasier, T., Dehoux, T. and Bertrand, A. (2012) Scaled behavior of interface waves at an imperfect solid-solid interface. Journal of Applied Physics, 112, 1-12.

[11] Wang, T. T., Hu, J. T., Chen, C. H. and Huang, T. H. (2014) Response of a tunnel in double layer rocks subjected to harmonic $\mathrm{P}$ and $\mathrm{S}$ waves. International Journal of Rock Mechanics and Mining Sciences, 70, 435-443.

[12] Huang, W., Wang, Y. J. and Rokhlin, S. I. (1996) Oblique scattering of an elastic wave from a multilayered cylinder in a solid. Journal of Acoustical Society of America, 99, 2742-2754. 
[13] Hasheminejad, S. M. and Rajabi, M. (2007) Acoustic resonance scattering from a submerged functionally graded cylindrical shell. Journal of Sound and Vibration, 302, 208-228.

[14] Shindo, Y. and Niwa, N. (1996) Scattering of antiplane shear waves in a fiber-reinforced composite medium with interfacial layers. Acta Mechanica, 117, 181-190.

[15] Li, F. M., Hu, C. and Huang, W. H. (2002) Scattering of elastic waves in an elastic matrix containing an inclusion with interfaces. Acta Mechanica Solida Sinica, 15, 270-276.

[16] Gleiter, H. (2000) Nanostructured Materials: Basic Concepts and Microstructure. Acta Materialia, 4, 1-29.

[17] Gurtin, M. E. and Murdoch, A. I. (1975) A continuum theory of elastic material surfaces. Archive for Rational Mechanics and Analysis, 57, 291-323.

[18] Gurtin, M. E., Weissmüller, J. and Larché, F. (1998) A general theory of curved deformable interfaces in solids at equilibrium. Philosophical Magazine A, 78, 1093-1109.

[19] Wang, G. F. and Wang, T. J. (2006) Surface effects on the diffraction of plane compressional waves by a nano-sized circular hole. Applied Physics Letters, 89, 1-3.

[20] Wang, G. F. (2007) Diffraction of plane compressional wave by a nano-sized spherical cavity with surface effects. Applied Physics Letters, 90, 1-3.
[21] Wang, G. F. (2009) Multiple diffraction of plane compressional waves by two circular cylindrical holes with surface effects. Journal of Applied Physics, 105, 1-6.

[22] Ru, Y., Wang, G. F. and Wang, T. J. (2009) Diffraction of elastic waves and stress concentration near a cylindrical nano-Inclusion incorporating surface effect. Journal of Vibration and Acoustics, 131, 1-7.

[23] Ou, Z. Y. and Lee, D. W. (2012) Effects of interface energy on multiple scattering of plane compressional waves by two cylindrical fibers. International Journal of Applied Mechanics, 4, 1-19.

[24] Ru, Y., Wang, G. F., Su, L. C. and Wang, T. J. (2013) Scattering of vertical shear waves by a cluster of nano-sized cylindrical holes with surface effect. Acta Mechanica, 224, 935-944.

[25] Ou, Z. Y. and Lee, D. W. (2012) Effects of interface energy on scattering of plane elastic wave by a nano-sized coated fiber. Journal of Sound and Vibration, 331, 5623-5643.

[26] Pao, Y. H. and Mow, C. C. (1973) Diffraction of elastic waves and dynamic stress concentrations. New York: Crane Russak \& Company.

[27] Fan, Z. F., Zhang J. C., Xu H. (2019) Theoretical study of the dynamic response of a circular lined tunnel with an imperfect interface subjected to incident $\mathrm{SV}$-waves. Computers and Geotechnics, 110, 308-318. 